\title{
Editorial
}

Cerebrovascular Diseases

\section{Risks of Acute Stroke Treatment in Patients with Cardiac Disease}

In this journal, Rice et al. [1] from USA present their study on 477 patients with left ventricular assist device: $10.3 \%$ suffered acute ischemic stroke, the majority (59\%) of which occurred in-hospital. Sixty-five per cent had INR values $<1.7$ and did not qualify for receiving iv thrombolysis. About 51\% underwent CT angiography and $33 \%$ showed arge vessel occlusions (mainly MCA occlusions) with $31 \%$ undergoing intra-arterial thrombectomy. A strong cooperation of interventional cardiologists and neurologists is important for managing this difficult and often underestimated common condition.

In addition, Holda and Koziej [2] from Poland present a meta-analysis on patients with a left-sided atrial septal pouch (LSSP) as an underestimated risk factor for stroke in patients otherwise classified as cryptogenic stroke. This condition is defined as a small, kangaroo pouch-like in- dentation of the human interatrial septum, associated with a 2 -fold increased risk of atrial fibrillation like a patent formen ovale, which is a communication across the inter-atrial septum. Seven studies (400 cryptogenic stroke patients and 1,456 non-stroke controls) were included in the meta-analysis. A total of 138 LSSPs were identified among the cryptogenic stroke patients, with a pooled prevalence of $29.8 \%$ (95\% CI 17.5-43.7\%), and 268 LSSPs were identified in the non-stroke controls, with a pooled prevalence of $21.0 \%$ (95\% CI $13.7-29.5 \%)$. The risk of cryptogenic stroke was higher in patients with an LSSP than in patients without LSSP (OR 1.52; 95\% CI 1.15$2.00 ; p<0.001)$. No significant heterogeneity was detected across the included studies $(p>0.05)$.

Michael G. Hennerici, Mannheim, Germany

\section{References}

1 Rice CJ, Cho S-M, Zhang LQ, Hassett C, Starling RC, Uchino K: The management of acute ischemic strokes and the prevalence of large vessel occlusion in left ventricular assist device. Cerebrovasc Dis 2018;46:213-217.
2 Holda MK, Koziej M: Left-sided atrial septal pouch as a risk factor of cryptogenic stroke: a systematic review and meta-analysis. Cerebrovasc Dis 2018;46:223-229. 